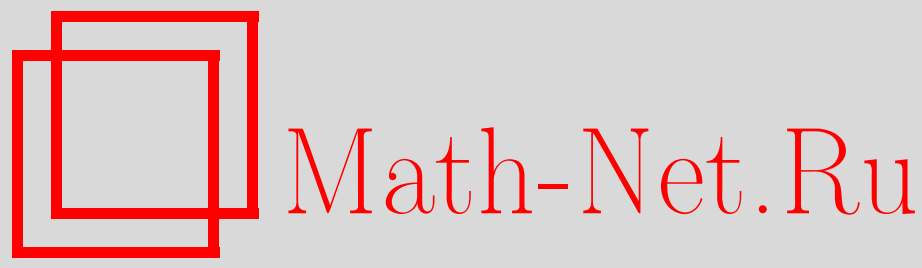

Н. В. Антонов, П. И. Какинь, Случайный рост границы раздела фаз в случайной среде: ренормгрупповой анализ простой модели, ТМФ, 2015, том 185, номер 1, 37-56

DOI: https://doi.org/10.4213/tmf8927

Использование Общероссийского математического портала Math-Net.Ru подразумевает, что вы прочитали и согласны с пользовательским соглашением http://www . mathnet.ru/rus/agreement

Параметры загрузки:

IP : 3.85 .5 .30

26 апреля 2023 г., $11: 34: 43$

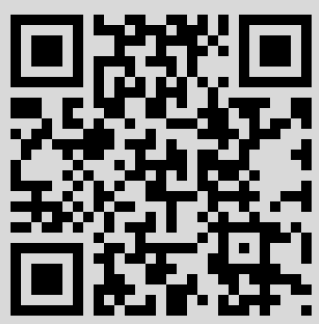




\title{
ФИЗИКА
}

Том 185, № 1

октябрь, 2015

\section{СЛУЧАЙНЫЙ РОСТ ГРАНИЦЫ РАЗДЕЛА ФАЗ В СЛУЧАЙНОЙ СРЕДЕ: РЕНОРМГРУППОВОЙ АНАЛИЗ ПРОСТОЙ МОДЕЛИ}

\begin{abstract}
Изучается влияние турбулентного перемешивания на случайный рост границы раздела фаз в задаче о выпадении осадка на подложку. Рост моделируется хорошо известной моделью Кардара-Паризи-Жанга. Турбулентное адвективное поле скорости моделируется ансамблем Крейчнана: гауссовой статистикой с корреляционной функцией $\langle v v\rangle \propto \delta\left(t-t^{\prime}\right) k^{-d-\xi}$, где $k-$ волновое число и $\xi-$ свободный параметр, $0<\xi<2$. Изучаются эффекты сжимаемости жидкости. С использованием теоретико-полевой группы перенормировок показано, что в зависимости от отношения показателя $\xi$ и пространственной размерности $d$ система демонстрирует различные типы крупномасштабного долговременного асимптотического поведения, связанного с четырьмя возможными фиксированными точками уравнения перенормировочной группы. Помимо уже известных режимов поведения (обыкновенная диффузия, обыкновенный процесс роста и пассивное адвективное скалярное поле), установлено существование нового неравновесного класса универсальности. Координаты фиксированных точек, их области устойчивости и критические размерности вычисляются в первом порядке двойного разложения по $\xi$ и $\varepsilon=2-d$ (однопетлевое приближение). Оказывается, что для несжимаемой жидкости наиболее реалистичные значения $\xi=4 / 3$ или $\xi=2$ и $d=1$ или $d=2$ соответствуют случаю пассивного скалярного поля, когда нелинейность модели Кардара-Паризи-Жанга несущественна и рост границы раздела фаз полностью определяются турбулентным переносом. Если сжимаемость приобретает достаточно большое значение, происходит изменение критического поведения, и эти значения $d$ и $\xi$ попадают в область устойчивости нового режима, где адвекция и нелинейность одинаково значимы. Тем не менее в этом режиме координаты фиксированной точки лежат в нефизической области, так что вопрос физической интерпретации остается открытым.
\end{abstract}

Ключевые слова: статистическая механика, критическое поведение, ренормгруппа, неравновесные системы, турбулентность.

DOI: $10.4213 /$ tmf8927

* Физический факультет, Санкт-Петербургский государственный университет, СанктПетербург, Россия. E-mail: n.antonov@spbu.ru, p.kakin@spbu.ru 


\section{1. ВВЕДЕНИЕ И ОПИСАНИЕ МОДЕЛИ}

В течение последних десятилетий процессы роста в различных физических системах вызывали неослабевающий интерес. Это фронты отвердевания и пламени, дым и коллоидные агрегаты, опухоли и т.п. (см., например, работы [1]-[9] и литературу в них). Наиболее характерный пример - выпадение осадка на подложку и рост соответствующей фазовой границы. Для описания этих явлений был выдвинут ряд микроскопических моделей: модели Идена [6], Эдвардса-Вилкинсона [7], ограниченные модели "solid-on-solid" [8], баллистическое выпадение [9]; и это еще не полный список.

Оказывается, однако, что все процессы роста имеют несколько важных общих черт с поведением равновесных почти критических систем: а именно самоподобное (скейлинговое) поведение (степенные зависимости) с достаточно универсальными (не зависящими от особенностей конкретного процесса) индексами. В частности, структурная функция порядка $n$ процесса роста ведет себя следующим образом $[1]-[5]:$

$$
S_{n}(t, r) \equiv\left\langle[h(t, \mathbf{x})-h(0, \mathbf{0})]^{n}\right\rangle \simeq r^{n \chi} F_{n}\left(t r^{z}\right), \quad r=|\mathbf{x}| .
$$

Здесь $h(x)=h(t, \mathbf{x})$ - высота профиля раздела, скобки $\langle\cdot\rangle$ обозначают усреднение по статистическому ансамблю, $\chi$ и $z$ - индекс жесткости и динамический индекс соответственно, а $F_{n}(\cdot)$ - определенная универсальная скейлинговая функция.

Отсюда естественным образом вытекает описание универсальных свойств процессов роста на основе определенных упрощенных моделей для сглаженного (крупнозернистого) поля высоты по аналогии с теорией критического состояния, где большинство типичных классов универсальности (типов критического поведения) описывается классической моделью $\varphi^{4}$ [10], [11]. В качестве крупнозернистой модели роста обычно используется модель Кадара-Паризи-Жанга (КПЖ) [12], описывающаяся нелинейным диференциальным уравнением

$$
\partial_{t} h=\varkappa_{0} \partial^{2} h+\lambda_{0} \frac{(\partial h)^{2}}{2}+f .
$$

Здесь поле высоты $h(x)=h(t, \mathbf{x})$ зависит от $d$-мерной координаты подложки $\mathbf{x}$, $\partial_{t}=\partial / \partial t, \partial_{i}=\partial / \partial x_{i}, \partial^{2}=\partial_{i} \partial_{i}-$ оператор Лапласа и $(\partial h)^{2}=\partial_{i} h \partial_{i} h$; суммирование по повторяющимся тензорным индексам везде подразумевается. Первый член в правой части уравнения (1.2) описывает поверхностное натяжение с коэффициентом $\varkappa_{0}>0$. Второй член представляет рост вдоль локальной нормали к поверхности. Параметр $\lambda_{0}$ может иметь любой знак; его можно убрать перемасштабированием, и в дальнейшем мы положим $\lambda_{0}=1$.

Далее, $f=f(x)$ - гауссов случайный шум с нулевым средним и заданной парной ковариацией ${ }^{1)}$

$$
\left\langle f(x) f\left(x^{\prime}\right)\right\rangle=2 D_{0} \delta\left(t-t^{\prime}\right) \delta^{(d)}\left(\mathbf{x}-\mathbf{x}^{\prime}\right)
$$

с положительным амплитудным множителем $D_{0}>0$.

Точнее говоря, модель (1.2), (1.3) появилась впервые в основополагающей статье Форстера, Нельсона и Стефена [13] в терминах чисто продольного (соленоидального) векторного поля $u_{i}=\partial_{i} h$. Тогда для $\lambda_{0}=-1$ уравнение (1.2) представляет собой

\footnotetext{
1) Строго говоря, для того чтобы исключить линейный по времени рост среднего значения $\langle h\rangle$, нужно ввести ненулевое среднее значение $\langle f\rangle$. Поскольку нас интересуют только величины типа (1.1), которые содержат только разности полей, обоими средними значениями можно одновременно пренебречь.
} 
$d$-мерное обобщение уравнения Бюргерса. Его также можно отобразить на модель ориентированных полимеров в случайной среде и на модель многочастичной бозесистемы с притяжением (см., например, статьи [14]).

Вообще первые два члена в правой части уравнения (1.2) - это всего лишь простейшие локальные члены, ковариантные по отношению к симметриям $h \rightarrow h+$ const и $O(d)$. Таким образом, модель КПЖ возникает повсеместно при описании многих неравновесных, неупорядоченных и диффузионных систем. Тогда поле $h$ можно трактовать по-разному. К примеру, в работах [15] модель КПЖ и ее следствия использовались для изучения крупномасштабного распределения вещества во Вселенной.

Были разработаны несколько обобщений и модификаций первоначальной формулировки модели КПЖ: случайный шум с конечным временем корреляции [16], векторное или матричное поле $h$ [17], измененная форма нелинейности [18] и анизотропная модификация [19]. В связи с последней стоит отметить непрерывные анизотропные модели самоорганизующейся критичности [20].

Теоретико-полевая перенормировочная группа (РГ) представляет собой мощную количественную теорию критического состояния (см. монографии [10], [11] и литературу в них). В РГ-подходе возможные классы универсальности связаны с ИКпритягивающими фиксированными точками перенормируемых теоретико-полевых моделей.

РГ-анализ модели КПЖ, начатый в работах [12], [13], в конечном итоге (после некоторого недопонимания) привел к следующим заключениям [21], [22]. В теоретико-полевой формулировке стохастическая задача (1.2), (1.3) мультипликативно перенормируема. Нелинейность $(\partial h)^{2}$ в (1.2) ИК-несущественна (в смысле Вильсона) для $d>2$, имеет логарифмический (пограничный) характер для $d=2$ и существенна для $d<2$. Таким образом, эту нелинейность можно изучать с помощью стандартной пертурбативной РГ и разложения по $\varepsilon \equiv 2-d$. Соответствующие уравнения РГ обладают нетривиальной фиксированной точкой с критическими индексами $\chi=0$, $z=2$ (точное соотношение $\chi+z=2$ продиктовано галилеевой симметрией). Тем не менее фиксированная точка для $\varepsilon<0$ является ИК-отталкивающей, тогда как для $\varepsilon>0$ она не лежит в физической области параметров модели $\left(D_{0}, \varkappa_{0}>0\right)$ и, таким образом, едва ли может описывать ИК-асимптотическое поведение задачи. Все эти результаты являются "пертурбативно точными", т. е. точными во всех порядках разложения по $\varepsilon$.

Тем не менее можно предположить, что модель КПЖ содержит гипотетическую ИК-притягивающую "сильно взаимодействующую” фиксированную точку, не проявляющуюся в рамках какой-либо теории возмущений. Тогда для $d=1$ флуктуационно-диссипационная теорема вместе с галилеевой симметрией дает точные значения $\chi=1 / 2, z=3 / 2$ [12], [13]. Сделав дополнительные (весьма нетривиальные) предположения, можно получить точные значения для критических индексов в случаях $d=2$ и $d=3$ [23]. Доказательство существования сильно взаимодействующей точки, обеспечивающееся так называемой функциональной (также известной как "точная", или "непертурбативная") РГ [24], [25], хотя и убедительно, количественно все еще недостаточно надежно, и саму ситуацию нельзя считать удовлетворительной. Некоторые другие открытые вопросы обсуждаются в работах [26], [27].

Хорошо известно, что поведение реальных систем вблизи критических точек крайне чувствительно к внешним воздействиям, гравитации, эффектам конечных 
размеров, присутствию примесей и т. п. (см., например, монографии [28], [29] для общего обсуждения и литературы). Более того, некоторые воздействия (случайно распределенные примеси в магнетиках и турбулентное перемешивание жидких систем) могут менять тип фазового перехода или порождать новые классы универсальности с богатым набором порой довольно необычных свойств.

Исследование влияния различных типов детерминированных или хаотических течений (ламинарных потоков, турбулентных конвекций и т. д.) на поведение критических систем (таких, как жидкие кристаллы или бинарные смеси в области точек смешивания) показало, что они могут разрушить обычное критическое поведение: оно может смениться на поведение типа среднего поля или, при выполнении определенных условий, перейти в более сложное поведение, описывающееся неравновесными классами универсальности [30]-[36].

В настоящей работе изучается влияние случайного (турбулентного) движения жидкости, содержащей растворенные частицы, на ИК-поведение случайно растущей границы раздела фаз, при этом обращается особое внимание на эффекты сжимаемости. Перенос полем скорости $\mathbf{v}(x) \equiv\left\{v_{i}(x)\right\}$ вводится "минимальной" заменой

$$
\partial_{t} h \rightarrow \nabla_{t} h \equiv \partial_{t} h+\left(v_{i} \partial_{i}\right) h,
$$

где $\nabla_{t}$ - галилеево-ковариантная (лагранжева) производная.

Мы собираемся предварительно понять на качественном уровне, что может произойти, если учесть движение жидкости. По этой причине мы пренебрегаем возможным влиянием поля $h(x)$ на динамику жидкости ("пассивная" адвекция) и моделируем поле скорости простой гауссовой статистикой с нулевым средним и заданной парной ковариацией с нулевым временем корреляции,

$$
\begin{gathered}
\left\langle v_{i}(t, \mathbf{x}) v_{j}\left(t^{\prime}, \mathbf{x}^{\prime}\right)\right\rangle=\delta\left(t-t^{\prime}\right) D_{i j}\left(\mathbf{x}-\mathbf{x}^{\prime}\right), \\
D_{i j}(\mathbf{r})=B_{0} \int_{k>m} \frac{d \mathbf{k}}{(2 \pi)^{d}} \frac{1}{k^{d+\xi}}\left\{P_{i j}(\mathbf{k})+\alpha Q_{i j}(\mathbf{k})\right\} e^{i \mathbf{k} \cdot \mathbf{r}},
\end{gathered}
$$

известной как ансамбль Казанцева-Крейчнана (см., например, статью [37]). Здесь $P_{i j}(\mathbf{k})=\delta_{i j}-k_{i} k_{j} / k^{2}$ и $Q_{i j}(\mathbf{k})=k_{i} k_{j} / k^{2}$ - поперечный и продольный проекторы соответственно, $k \equiv|\mathbf{k}|$ - волновое число, $B_{0}>0$ - амплитудный множитель, $\alpha>0$ - произвольный параметр. Случай $\alpha=0$ соответствует несжимаемой жидкости $\left(\partial_{i} v_{i}=0\right)$, тогда как предел $\alpha \rightarrow \infty$ при фиксированном $\alpha B_{0}$ соответствует чисто потенциальному полю скорости. Показатель $\xi$ - свободный параметр, $0<\xi<2$, его можно рассматривать как своего рода показатель Гёльдера, который характеризует "негладкость" поля скорости; “колмогоровское" значение - это $\xi=4 / 3$, тогда как "бэтчелоровский" предел $\xi \rightarrow 2$ отвечает случаю очень гладкой скорости. Обрезание в интеграле $(1.5)$ снизу на $k=m$, где $m \equiv 1 / \mathcal{L}$, отвечает интегральному масштабу турбулентности $\mathcal{L}$, обеспечивая ИК-регуляризацию. Его точная форма не важна; резкое обрезание - простейший выбор из практических соображений.

Этот ансамбль, хотя он и кажется простым, привлек огромное внимание при исследовании турбулентности из-за того, что он дает глубокое понимание происхождения перемежаемости и аномального мультискейлинга в турбулентной адвекции и турбулентности в целом (см. обзор [37] и литературу в нем). РГ-подход к этой задаче обсуждается в работе [38]. В контексте нашего исследования ансамбль Казанцева-Крейчнана особенно полезен тем, что он позволяет без особого труда моделировать сжимаемость, в то время как, если описывать поле скорости 
уравнениями Навье-Стокса (см., например, работы [39], [40]), это довольно сложно делать. Для сжимаемого поля $\left(\partial_{i} v_{i} \neq 0\right)$ ковариантную производную можно ввести другим способом, а именно как $\nabla_{t} h \equiv \partial_{t} h+\partial_{i}\left(v_{i} h\right)$, что является обязательным, если под полем $h$ подразумевается плотность некоторой сохраняющейся величины. В нашем случае, однако, $h$ "не сохраняется" из-за нелинейного члена в (1.2), и в дальнейшем мы будем расматривать только вариант (1.4), потому что он не нарушает симметрию $h \rightarrow h+$ const первоначальной модели КПЖ.

План работы следующий. В разделе 2 представлена теоретико-полевая формулировка полной стохастической задачи (1.2), (1.3), (1.5) и диаграммная техника. В разделе 3 мы анализируем УФ-расходимости модели и показываем ее мультипликативную перенормируемость. Тогда уравнения РГ, как и уравнения критического скейлинга, можно получить стандартным образом (см. раздел 4). Фиксированные точки уравнений РГ и возможные масштабные режимы изучаются в разделе 5 . Оказывается, что в дополнение к гауссовой фиксированной точке (свободная теория поля), чисто "кинематическому" режиму (нелинейность КПЖ несущественна в смысле Вильсона) и фиксированной точке чистой модели КПЖ (турбулентный перенос несуществен) уравнения РГ содержат полностью нетривиальную фиксированную точку, в которой и нелинейность, и перемешивания важны. Соответствующие критические индексы можно вычислить с помощью двойного разложения по $\xi$ и $\varepsilon=2-d$; мы выводим их в ведущем однопетлевом порядке.

В работе найдены области ИК-устойчивости фиксированных точек в пространстве параметров $\varepsilon, \xi$ и $\alpha$. В частности, оказывается, что для малых $\alpha$ и наиболее реалистичных значений $d=1$ или $d=2$ и $\xi=4 / 3$ или $\xi=2$ ИК-асимптотическое поведение управляется кинематической фиксированной точкой с точно известными критическими индексами. По мере роста степени сжимаемости $\alpha$ область устойчивости полномасштабной точки расширяется и, наконец, достигает реалистичных значений $\varepsilon$ и $\xi$.

Вывод выражений типа (1.1) требует (довольно простого) анализа составного поля $h^{n}(x)$; он обсуждается в разделе 6 .

Следует признать, что, хотя полученные практические результаты выводятся в рамках стандартной “пертурбативной" теоретико-полевой РГ, сильно взаимодействующая ИК-притягивающая фиксированная точка, если она действительно существует, определенно выживает и в полномасштабной модели, но вопрос ее ИКустойчивости выходит за рамки нашего исследования. Этот вопрос наряду с некоторыми другими обсуждается в разделе 7 .

Практические вычисления перенормировочных констант и РГ-функций приведены в приложении.

\section{2. ТЕОРЕТИКО-ПОЛЕВАЯ ФОРМУЛИРОВКА МОДЕЛИ}

Рассмотрим сначала исходную модель КПЖ без адвекции. Согласно общему утверждению [41] (см. также монографии [10], [11]) стохастическая задача (1.2), (1.3) эквивалентна теоретико-полевой модели двух полей $\Phi=\left\{h, h^{\prime}\right\}$ с функционалом действия

$$
\mathcal{S}(\Phi)=\frac{1}{2} h^{\prime} D_{0} h^{\prime}+h^{\prime}\left\{-\partial_{t} h+\varkappa_{0} \partial^{2} h+\frac{1}{2}(\partial h)^{2}\right\}
$$


(мы положили $\left.\lambda_{0}=1\right)$. Здесь и далее все необходимые интегрирования по переменной $x=(t, \mathbf{x})$ и суммирования по повторяющимся тензорным индексам подразумеваются, например

$$
h^{\prime} D_{0} h^{\prime}=D_{0} \int d t \int d \mathbf{x} h^{\prime}(t, \mathbf{x}) h^{\prime}(t, \mathbf{x}) .
$$

Теоретико-полевая формулировка означает, что различные корреляционные функции и функции отклика стохастической задачи (1.2), (1.3) могут быть соотнесены с различными функциями Грина теоретико-полевой модели с действием (2.1). Иными словами, они представляются функциональными средними по полному набору полей $\Phi=\left\{h, h^{\prime}\right\}$ с весом $\exp \mathcal{S}(\Phi)$.

Затравочные пропагаторы в соответствующей диаграммной технике Фейнмана определяются свободными (билинейными по полям) частями действия (2.1). В частотно-импульсном $(\omega-\mathbf{k})$ представлении они имеют вид

$$
\left\langle h h^{\prime}\right\rangle_{0}=\left\langle h^{\prime} h\right\rangle_{0}^{*}=\frac{1}{-\mathrm{i} \omega+\varkappa_{0} k^{2}}, \quad\langle h h\rangle_{0}=\frac{D_{0}}{\omega^{2}+\varkappa_{0}^{2} k^{4}}, \quad\left\langle h^{\prime} h^{\prime}\right\rangle_{0}=0 .
$$

В этой модели есть только одна вершина взаимодействия, а именно $h^{\prime}(\partial h)^{2} / 2$.

В диаграммном представлении мы обозначаем $\langle h h\rangle_{0}$ прямой линией, а $\left\langle h h^{\prime}\right\rangle_{0}-$ прямой линией со штрихом, соответствующим полю $h^{\prime}$.

Включение поля скорости $\mathbf{v}(x) \equiv\left\{v_{i}(x)\right\}$ выполняется путем замены (1.4) в (1.2) и, таким образом, в (2.1). Полная задача тогда становится эквивалентной теоретикополевой модели трех полей $\Phi=\left\{h, h^{\prime}, \mathbf{v}\right\}$ с функционалом действия

$$
\mathcal{S}(\Phi)=\frac{1}{2} h^{\prime} D_{0} h^{\prime}+h^{\prime}\left\{-\nabla_{t} h+\varkappa_{0} \partial^{2} h+\frac{1}{2}(\partial h)^{2}\right\}+\mathcal{S}_{\mathbf{v}} .
$$

Последний член отвечает гауссову усреднению по полю $\mathbf{v}$ с коррелятором (1.5):

$$
\mathcal{S}_{\mathbf{v}}=-\frac{1}{2} \int d t \int d \mathbf{x} \int d \mathbf{x}^{\prime} v_{i}(t, \mathbf{x}) D_{i j}^{-1}\left(\mathbf{x}-\mathbf{x}^{\prime}\right) v_{j}\left(t, \mathbf{x}^{\prime}\right),
$$

где $D_{i j}^{-1}$ - оператор, обратный к оператору $D_{i j}$ из (1.5).

Таким образом, диаграммы Фейнмана для полной модели (2.4) включают, в дополнение к (2.3), новый пропагатор (1.5) и новую вершину $-h^{\prime}(v \partial) h$.

Роль констант взаимодействия в обыкновенной теории возмущений играют два параметра

$$
g_{0}=\frac{D_{0}}{\varkappa_{0}^{3}} \sim \Lambda^{\varepsilon}, \quad w_{0}=\frac{B_{0}}{\varkappa_{0}} \sim \Lambda^{\xi} .
$$

Последние соотношения вытекают из соображений размерности (см. следующий раздел) и определяют характерный импульсный УФ-масштаб $\Lambda$.

\section{3. УФ-РАСХОДИМОСТИ И ПЕРЕНОРМИРОВКА}

Хорошо известно, что анализ УФ-расходимостей основывается на анализе канонических размерностей (“подсчете степеней"), см., например, монографии [10], [11]. Динамические модели типа (2.4) имеют два независисмых масштаба: временной масштаб $T$ и масштаб длины $L$ (в отличие от одномасштабных статических моделей). Таким образом, каноническая размерность некоторой величины $F$ (поля или параметра в функционале действия) может быть полностью описана двумя числами, частотной размерностью $d_{F}^{\omega}$ и импульсной размерностью $d_{F}^{k}$ :

$$
[F] \sim[T]^{-d_{F}^{\omega}}[L]^{-d_{F}^{k}} .
$$


Они находятся из очевидных условий нормировки

$$
d_{k}^{k}=-d_{\mathbf{x}}^{k}=1, \quad d_{k}^{\omega}=d_{\mathbf{x}}^{\omega}=0, \quad d_{\omega}^{k}=d_{t}^{k}=0, \quad d_{\omega}^{\omega}=-d_{t}^{\omega}=1
$$

и из требования, чтобы каждый член функционала действия был безразмерным (по отношению к импульсной и частотной размерностям отдельно). Тогда, основываясь на размерностях $d_{F}^{k}$ и $d_{F}^{\omega}$, можно ввести полную каноническую размерность $d_{F}=$ $d_{F}^{k}+2 d_{F}^{\omega}$ (в свободной теории $\partial_{t} \propto \partial^{2}$ ). В теории перенормировки динамических моделей эта величина играет ту же роль, что и привычная (импульсная) размерность в статических задачах (см. книгу [11], гл. 5).

Канонические размерности полей и параметров модели (2.4) приведены в табл. 1. В ней также представлены перенормированные параметры (без нижнего индекса 0) и перенормировочная масса $\mu$, которая будет введена ниже.

ТАБлицА 1. Канонические размерности полей и параметров в модели (2.4).

\begin{tabular}{|c|r|c|r|c|c|c|c|c|c|c|}
\hline$F$ & $h$ & $h^{\prime}$ & $\mathbf{v}$ & $\varkappa_{0}, \varkappa$ & $D_{0}$ & $g_{0}$ & $B_{0}$ & $w_{0}$ & $g, w, \alpha$ & $m, \mu, \Lambda$ \\
\hline$d_{F}^{\omega}$ & 1 & -1 & 1 & 1 & 3 & 0 & 1 & 0 & 0 & 0 \\
\hline$d_{F}^{k}$ & -2 & $d+2$ & -1 & -2 & $-d-4$ & $2-d \equiv \varepsilon$ & $-2+\xi$ & $\xi$ & 0 & 1 \\
\hline$d_{F}$ & 0 & $d$ & 1 & 0 & $\varepsilon$ & $\varepsilon$ & $\xi$ & $\xi$ & 0 & 1 \\
\hline
\end{tabular}

Из табл. 1 следует, что модель имеет логарифмический характер при $d=2$ и $\xi=0$, когда обе константы взаимодействия $g_{0}$ и $w_{0}$ одновременно становятся безразмерными. Отсюда УФ-расходимости в функциях Грина предстают в виде полюсов по $\varepsilon=2-d, \xi$ и, в общем случае, во всех их линейных комбинациях.

Полная каноническая размерность произвольной 1-неприводимой функции Грина $\Gamma=\langle\Phi \ldots \Phi\rangle_{1 \text {-непр }}$, где $\Phi=\left\{h, h^{\prime}, v\right\}$, в частотно-импульсном представлении дается соотношением

$$
d_{\Gamma}=d+2-d_{h} N_{h}-d_{h^{\prime}} N_{h^{\prime}}-d_{v} N_{v}
$$

где $N_{h}, N_{h^{\prime}}, N_{v}$ - числа соответствующих полей, входящих в функцию $Г$ (см., например, [11]).

Полная размерность $d_{\Gamma}$ в логарифмической теории (т. е. при $\varepsilon=\xi=0$ ) - это формальный индекс УФ-расходимости: $\delta_{\Gamma}=\left.d_{\Gamma}\right|_{\varepsilon=\xi=0}$. Поверхностная УФ-расходимость, для исключения которой нужны контрчлены, может присутствовать только в тех функциях $Г$, для которых $\delta_{\Gamma}$ есть неотрицательное целое число. Контрчлен это полином по частотам и импульсам степени $\delta_{\Gamma}$ (при условии, что подразумевается соглашение $\left.\omega \propto k^{2}\right)$.

Если по какой-либо причине некоторое число внешних импульсов возникает как общий множитель во всех диаграммах данной функции Грина, то реальный индекс расходимости $\delta_{\Gamma}^{\prime}$ будет меньше, чем $\delta_{\Gamma}$, на соответствующее число единиц. Именно это и происходит в нашей модели: поле $h$ входит в вершины $h^{\prime}(\partial h)^{2}$ и $h^{\prime}(v \partial) h$ только в форме пространственной производной. Поэтому любое появление $h$ в какой-либо функции Г приводит к такому внешнему импульсу, и реальный индекс расходимости дается выражением $\delta_{\Gamma}^{\prime}=\delta_{\Gamma}-N_{h}$. Более того, $h$ может возникнуть в соответствующем контрчлене только в форме производной.

Из табл. 1 и выражения (3.1) получаем

$$
\delta_{\Gamma}^{\prime}=\delta_{\Gamma}-N_{h}=4-N_{h}-2 N_{h^{\prime}}-N_{v} .
$$


Рис. 1. Диаграмма исчезающей функции $\langle h h\rangle_{1-н е п р ~}$ с замкнутым циклом из двух запаздывающих пропагаторов.

В динамических моделях все 1-неприводимые функции без полей отклика тождественно равны нулю (их диаграммы всегда содержат замкнутые циклы запаздывающих линий), см., например, [11]. Пример такой диаграммы изображен на рис. 1. Таким образом, в (3.2) достаточно рассмотреть случай $N_{h^{\prime}}>0$.

Простой анализ выражения (3.2) показывает, что поверхностные УФ-расходимости могут присутствовать только в следующих 1-неприводимых функциях:

$$
\begin{array}{lllll}
\left\langle h^{\prime} h^{\prime}\right\rangle_{1 \text {-непр }} & \left(\delta_{\Gamma}=0,\right. & \left.\delta_{\Gamma}^{\prime}=0\right) & \text { с контрчленом } & h^{\prime} h^{\prime}, \\
\left\langle h^{\prime} h h\right\rangle_{1-\text {-нер }} & \left(\delta_{\Gamma}=2,\right. & \left.\delta_{\Gamma}^{\prime}=0\right) & \text { с контрчленом } & h^{\prime}(\partial h)^{2}, \\
\left\langle h^{\prime} h\right\rangle_{1 \text {-непр }} & \left(\delta_{\Gamma}=2,\right. & \left.\delta_{\Gamma}^{\prime}=1\right) & \text { с контрчленом } & h^{\prime} \partial^{2} h, \\
\left\langle h^{\prime} h v\right\rangle_{1-\text {-нер }} & \left(\delta_{\Gamma}=1,\right. & \left.\delta_{\Gamma}^{\prime}=0\right) & \text { с контрчленом } & h^{\prime}(v \partial) h, \\
\left\langle h^{\prime}\right\rangle_{1 \text {-непр }} & \left(\delta_{\Gamma}=2,\right. & \left.\delta_{\Gamma}^{\prime}=2\right) & \text { с контрчленом } & h^{\prime}, \\
\left\langle h^{\prime} v\right\rangle_{1 \text {-непр }} & \left(\delta_{\Gamma}=1,\right. & \left.\delta_{\Gamma}^{\prime}=1\right) & \text { с контрчленом } & h^{\prime}(\partial v), \\
\left\langle h^{\prime} v v\right\rangle_{1-\text {-нер }} & \left(\delta_{\Gamma}=0,\right. & \left.\delta_{\Gamma}^{\prime}=0\right) & \text { с контрчленом } & h^{\prime} v^{2} .
\end{array}
$$

Некоторые дополнительные соображения сокращают число необходимых контрчленов.

Действие модели КПЖ инвариантно относительно преобразования

$$
h(t, \mathbf{x}) \rightarrow h(t, \mathbf{x}+\mathbf{u} t)-\mathbf{u} \cdot \mathbf{x}, \quad h^{\prime}(t, \mathbf{x}) \rightarrow h^{\prime}(t, \mathbf{x}+\mathbf{u} t)
$$

с произвольным постоянным параметром $\mathbf{u}$. Эта инвариантность, которая становится галилеевой в терминах векторного поля $\partial_{i} h$, нарушается в полной модели (2.1). Тем не менее в последней имеется другая галилеева симметрия, а именно

$$
\begin{gathered}
h(t, \mathbf{x}) \rightarrow h(t, \mathbf{x}+\mathbf{u} t), \quad h^{\prime}(t, \mathbf{x}) \rightarrow h^{\prime}(t, \mathbf{x}+\mathbf{u} t), \\
\mathbf{v}(t, \mathbf{x}) \rightarrow \mathbf{v}(t, \mathbf{x}+\mathbf{u} t)-\mathbf{u}
\end{gathered}
$$

(здесь важно, что наше поле скорости некоррелировано во времени). Эта симметрия требует, чтобы моном $h^{\prime}(v \partial) h$ входил в контрчлен только в форме инвариантной комбинации $h^{\prime} \nabla_{t} h=h^{\prime} \partial_{t} h+h^{\prime}(v \partial) h$. Первый член тем не менее запрещен реальным индексом (3.2): поле $h$ появляется там без пространственной производной. Таким образом, и второй член оказывается под запретом (сокращение расходящихся вкладов от разных диаграмм может быть также проверено вручную). Кроме того, требование галилеевой симметрии исключает моном $h^{\prime} v^{2}$.

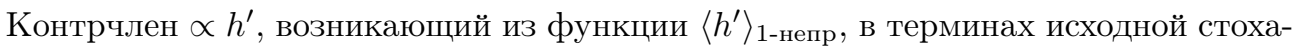
стической задачи перенормирует среднее значение случайного шума $\langle f\rangle$. Это можно проиллюстрировать диаграммой "головастик" на рис. 2: она представляет однопетлевой вклад в среднее значение $\langle\partial h \partial h\rangle$. Как уже было сказано (см. сноску на стр. 38), эти два средних значения можно одновременно игнорировать. В этом смысле вклад от функции $\left\langle h^{\prime}\right\rangle_{1-н е п р ~}$ схож со сдвигом критической температуры в моделях равновесного критического поведения. 


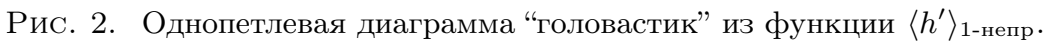

Контрчлен вида $h^{\prime} \partial_{i} v_{i}$, возникающий от функции Грина $\left\langle h^{\prime} v\right\rangle_{1 \text {-непр }}$, также требует особого обсуждения. Он полностью исчезает в случае несжимаемой жидкости, когда $\partial_{i} v_{i}=0$. Тем не менее практическое однопетлевое вычисление показывает, что он отсутствует и в общем случае $\alpha \neq 0$. Можно выдвинуть некоторые соображения, что это справедливо во всех порядках теории возмущений (см. приложение). Так как наш текущий анализ ограничивается однопетлевыми вычислениями, мы не будем в дальнейшем учитывать этот член.

Тогда остаются три контрчлена вида $h^{\prime} h^{\prime}, h^{\prime} \partial^{2} h$ и $h^{\prime}(\partial h)^{2}$. Все эти члены присутствуют в действии (2.4), делая нашу модель мультипликативно перенормируемой. Перенормированное действие может быть записано в форме

$$
\mathcal{S}_{\mathrm{R}}(\Phi)=\frac{1}{2} Z_{1} h^{\prime} D h^{\prime}+h^{\prime}\left\{-\partial_{t} h+\varkappa Z_{2} \partial^{2} h+\frac{1}{2} Z_{3}(\partial h)^{2}\right\}+\mathcal{S}_{\mathbf{v}},
$$

где $g, w$ и $\varkappa$ - перенормированные аналоги затравочных параметров и функционал $\mathcal{S}_{\mathbf{v}}$ из $(2.5)$ следует также выразить через перенормированные переменные. Перенормировочные константы $Z_{i}$ зависят только от полностью безразмерных параметров $g, w, \varkappa, \alpha$ и поглощают все полюсы по $\varepsilon$ и $\xi$.

Перенормированное действие (3.6) получается из исходного (2.4) перенормировкой полей $h \rightarrow Z_{h} h, h^{\prime} \rightarrow Z_{h^{\prime}} h^{\prime}$ и заменой параметров

$$
\varkappa_{0}=\varkappa Z_{\varkappa}, \quad g_{0}=g \mu^{\varepsilon} Z_{g}, \quad w_{0}=w \mu^{\xi} Z_{w} .
$$

Амплитуды $D$ и $B$ выражаются через перенормированные параметры следующим образом:

$$
D=g \varkappa^{3} \mu^{\varepsilon}, \quad B=w \varkappa \mu^{\xi} .
$$

Перенормировочные константы в уравнениях (3.6) и (3.7) связаны уравнениями

$$
\begin{gathered}
Z_{g}=Z_{1} Z_{2}^{-3} Z_{3}^{2}, \quad Z_{\varkappa}=Z_{2}, \quad Z_{h}=Z_{3}, \quad Z_{h^{\prime}}=Z_{3}^{-1}, \\
Z_{h} Z_{h^{\prime}}=1, \quad Z_{v}=1, \quad Z_{w} Z_{\varkappa}=1 .
\end{gathered}
$$

Первые два соотношения во второй строчке возникают из-за отсутствия контрчлена $h^{\prime} \nabla_{t} h$, последнее вытекает из отсутствия перенормировки члена $\mathcal{S}_{v}$.

Перенормировочные константы $Z_{1}, Z_{2}$ и $Z_{3}$ вычисляются непосредственно из диаграмм, затем константы в (3.7) находятся из соотношений (3.9). Перенормировочные константы можно найти из требования, чтобы функции Грина перенормированной модели (3.6) были УФ-конечны, после того как мы выразим их через перенормированные переменные. В нашем случае это означает, что функции Грина УФ-конечны при $\varepsilon, \xi \rightarrow 0$. Вычисление в первом порядке по $g$ и $w$ (однопетлевое приближение) дает (см. приложение для деталей)

$$
Z_{1}=1-\frac{1}{8 \varepsilon} \hat{g}-\frac{\alpha}{2 \xi} \hat{w}, \quad Z_{2}=Z_{3}=1-\frac{\hat{w}}{\xi} \frac{d-1+\alpha}{2 d},
$$

где

$$
\hat{g}=g \frac{S_{d}}{(2 \pi)^{d}}, \quad \hat{w}=w \frac{S_{d}}{(2 \pi)^{d}}
$$


здесь $S_{d}=2 \pi^{d} / \Gamma(d / 2)$ - площадь поверхности единичной сферы в пространстве размерности $d$.

Если использовать схему минимальных вычитаний (MB), то перенормировочные константы принимают вид $Z=1+$ полюсы только по $\varepsilon$ и $\xi$ (и в высших порядках - по их линейным комбинациям). Тогда, строго говоря, в условиях нашего однопетлевого приближения мы должны сделать замену $d=2-\varepsilon \rightarrow 2$ в выражениях выше. Однако некоторое время мы продолжим использовать их в форме (3.10), тогда можно будет получить некоторые точные результаты (см. раздел 5). Похожая перенормировочная схема, где размерность $d$ удерживается в "геометрических множителях", возникающих из сверток различных проекторов, ранее использовалась в работе [21]; ее справедливость и эквивалентность схеме МВ продемонстрирована в статье [42].

\section{4. УРАВНЕНИЯ РГ И РГ-ФУНКЦИИ}

Кратко рассмотрим простой вывод уравнений РГ; детальное рассмотрение можно найти в монографиях [10], [11]. Уравнения РГ пишутся для перенормированных функций Грина $G_{\mathrm{R}}=\langle\Phi \ldots \Phi\rangle_{\mathrm{R}}$. Они отличаются от исходных (неперенормированных) функций Грина $G$ общими численными множителями (из-за перемасштабирования полей) и другим выбором параметров $\left(e, \mu\right.$ вместо $\left.e_{0}\right)$. Таким образом, перенормированные функции Грина также можно использовать для анализа критического поведения. Связь $S_{\mathrm{R}}\left(Z_{\Phi} \Phi, e, \mu\right)=S\left(\Phi, e_{0}\right)$ между функционалами $(2.4)$ и (3.6) приводит к соотношениям

$$
G\left(e_{0}, \ldots\right)=Z_{h}^{N_{h}} Z_{h^{\prime}}^{N_{h^{\prime}}} G_{\mathrm{R}}(e, \mu, \ldots)
$$

между функциями Грина. Здесь, как и выше, $N_{h}$ и $N_{h^{\prime}}$ - числа соответствующих полей, входящих в $G$ (мы учитываем, что в нашей модели $\left.Z_{v}=1\right), e_{0}=\left\{g_{0}, \varkappa_{0}, w_{0}\right\}-$ полный набор затравочных параметров и $e=\{g, \varkappa, w\}$ - их перенормированные эквиваленты; многоточие обозначает остальные аргументы (время, координаты, импульсы).

Мы используем символ $\widetilde{\mathcal{D}}_{\mu}$ для обозначения дифференциального оператора $\left.\mu \partial_{\mu}\right|_{e_{0}}$ при фиксированных затравочных параметрах. Если выразить его через перенормированные переменные, он будет выглядеть следующим образом:

$$
\mathcal{D}_{\mathrm{RG}} \equiv \mathcal{D}_{\mu}+\beta_{g} \partial_{g}+\beta_{w} \partial_{w}-\gamma_{\varkappa} \mathcal{D}_{\varkappa},
$$

где $\mathcal{D}_{x} \equiv x \partial_{x}$ для любой переменной $x$. Аномальные размерности $\gamma$ определяются как

$$
\gamma_{F} \equiv \widetilde{\mathcal{D}}_{\mu} \ln Z_{F} \quad \text { для любой величины } F \text {, }
$$

а $\beta$-функции для двух безразмерных констант взаимодействия $g$ и $w$ таковы:

$$
\beta_{g} \equiv \widetilde{\mathcal{D}}_{\mu} g=g\left[-\varepsilon-\gamma_{g}\right], \quad \beta_{w} \equiv \widetilde{\mathcal{D}}_{\mu} w=w\left[-\xi-\gamma_{w}\right],
$$

где вторые равенства в каждой из формул вытекают из определений и соотношений (3.7).

Чтобы вывести основные дифференциальные уравнения РГ, мы применяем к обеим частям равенства $(4.1)$ оператор $\widetilde{\mathcal{D}}_{\mu}$ :

$$
\left\{\mathcal{D}_{\mathrm{RG}}+N_{h} \gamma_{h}+N_{h^{\prime}} \gamma_{h^{\prime}}\right\} G_{\mathrm{R}}(e, \mu, \ldots)=0 .
$$

Наконец, уравнения (3.9) приводят к следующим соотношениям между аномальными размерностями (4.3):

$$
\gamma_{h}=\gamma_{3}, \quad \gamma_{h^{\prime}}=-\gamma_{3}, \quad \gamma_{w}=-\gamma_{\varkappa}, \quad \gamma_{v}=1, \quad \gamma_{\varkappa}=\gamma_{2}, \quad \gamma_{g}=\gamma_{1}-3 \gamma_{2}+2 \gamma_{3} .
$$


Аномальная размерность, соответствующая данной перенормировочной константе $Z_{F}$, может быть найдена из соотношения

$$
\gamma_{F}=\left(\beta_{g} \partial_{g}+\beta_{w} \partial_{w}\right) \ln Z_{F} \simeq-\left(\varepsilon \mathcal{D}_{g}+\xi \mathcal{D}_{w}\right) \ln Z_{F}
$$

которое получается из определения (4.3), выражения (4.2) для оператора $\widetilde{\mathcal{D}}_{\mu}$ в перенормированных переменных и того факта, что перенормировочные константы зависят только от двух полностью безразмерных констант взаимодействия $g$ и $w$. Во второй части соотношения (4.7) мы оставили в $\beta$-функциях (4.4) только члены старшего порядка, так как их достаточно для однопетлевого приближения. Используя схему МВ в однопетлевом приближении, из явных выражений (3.10) получаем

$$
\gamma_{1}=\frac{\hat{g}}{8}+\frac{\alpha \hat{w}}{2}, \quad \gamma_{2}=\gamma_{3}=\hat{w} \frac{d-1+\alpha}{2 d},
$$

где $\hat{g}$ и $\hat{w}$ были определены ранее в (3.10), а поправки имеют порядок $\hat{g}^{2}, \hat{w}^{2}, \hat{w} \hat{g}$ и выше.

\section{5. ФИКСИРОВАННЫЕ ТОЧКИ И СКЕЙЛИНГОВЫЕ РЕЖИМЫ}

Хорошо известно, что асимптотическое поведение (при больших временах и расстояниях) перенормируемой теории поля определяется ИК-притягивающими фиксированными точками соответствующих уравнений РГ. В общем случае координаты возможных фиксированных точек находятся из требования, чтобы все $\beta$-функции обратились в нуль. В модели (2.4) координаты $g_{*}, w_{*}$ определяются двумя уравнениями

$$
\beta_{g}\left(g_{*}, w_{*}\right)=0, \quad \beta_{w}\left(g_{*}, w_{*}\right)=0,
$$

где $\beta$-функции берутся из (4.4). Тип фиксированной точки определяется матрицей

$$
\Omega=\left\{\Omega_{i j}=\frac{\partial \beta_{i}}{\partial g_{j}}\right\}
$$

где $\beta_{i}$ - полный набор $\beta$-функций и $g_{j}=\{g, w\}$ - полный набор констант взаимодействия. Для ИК-притягивающей фиксированной точки матрица $\Omega$ должна быть положительно определенной, т. е. действительные части всех ее собственных чисел должны быть положительными.

Из соотношений (4.6) мы находим, что $\gamma_{g}=\gamma_{1}-\gamma_{2}, \gamma_{w}=-\gamma_{\varkappa}=\gamma_{2}$, так что

$$
\beta_{g}=g\left(-\varepsilon-\gamma_{g}\right)=g\left(-\varepsilon-\gamma_{1}+\gamma_{2}\right), \quad \beta_{w}=w\left(-\xi-\gamma_{w}\right)=w\left(-\xi-\gamma_{2}\right) .
$$

Подставляя явные выражения (4.8), мы приходим к явным однопетлевым выражениям для $\beta$-функций:

$$
\beta_{g}=g\left\{-\varepsilon-\frac{\hat{g}}{8}+\hat{w} \frac{(d-1)(\alpha-1)}{2 d}\right\}, \quad \beta_{w}=w\left\{-\xi+\hat{w} \frac{d-1+\alpha}{2 d}\right\},
$$

где возможны поправки порядка $w^{2}$ и выше. Стоит отметить, что однопетлевой результат (5.4) для $\beta_{w}$ становится точным при $g=0$ (см., например, работу [38]), тогда как результат для $\beta_{g}$ становится точным при $w=0$, как следует из анализа в статьях [22].

Из уравнений (5.1) и (5.3) следует, что в нашей модели имеются четыре фиксированные точки. Так как $\partial_{g} \beta_{w}=0$, матрица $\Omega$ треугольная в каждом случае, и ее собственные числа просто даются диагональными элементами $\Omega_{g}=\partial \beta_{g} / \partial g$ и $\Omega_{w}=\partial \beta_{w} / \partial w$. Перечислим эти фиксированные точки. 


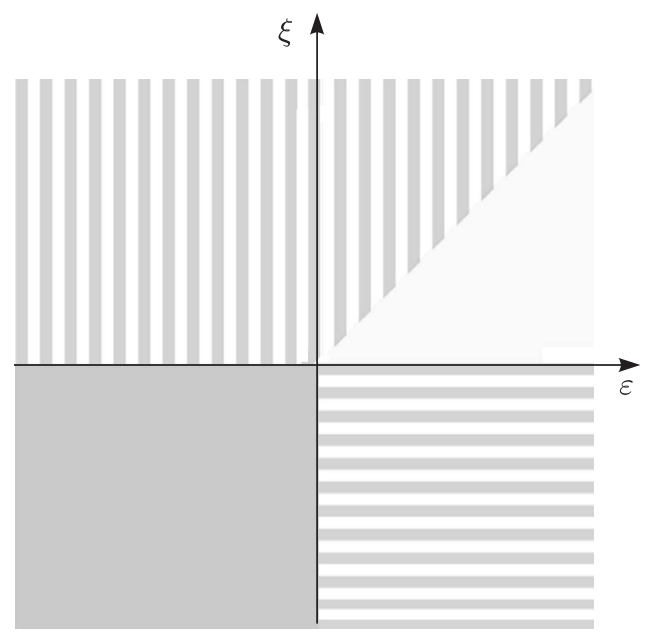

Рис. 3. Области устойчивости фиксированных точек модели (2.4).

1. Гауссова (свободная) фиксированная точка такая, что $g_{*}=w_{*}=0, \Omega_{g}=-\varepsilon$, $\Omega_{w}=-\xi$ (все эти выражения точные).

2. Фиксированная точка, для которой $w_{*}=0$ (точный результат во всех порядках), $\hat{g}_{*}=-8 \varepsilon, \Omega_{g}=\varepsilon, \Omega_{w}=-\xi$. Эта фиксированная точка соответствует чистой модели КПЖ: хотя имеется взаимодействие с полем скорости, оно не влияет на старший порядок ИК-асимптотического поведения (оно несущественно в смысле Вильсона).

3. Фиксированная точка, для которой $g_{*}=0$ (точный результат),

$$
\hat{w}_{*}=\frac{2 d \xi}{d-1+\alpha}, \quad \Omega_{g}=-\varepsilon+\xi-\frac{d \alpha \xi}{d-1+\alpha}
$$

и $\Omega_{w}=\xi$ (точный результат). Эта фиксированная точка соответствует чистой модели Крейчнана с маломасштабным перемешиванием; нелинейность КПЖ несущественна в смысле Вильсона.

4. Фиксированная точка, для которой

$$
\begin{array}{cc}
\hat{g}_{*}=8\left\{-\varepsilon+\xi-\frac{d \alpha \xi}{d-1+\alpha}\right\}, & \hat{w}_{*}=\frac{2 d \xi}{d-1+\alpha}, \\
\Omega_{g}=\varepsilon-\xi+\frac{d \alpha \xi}{d-1+\alpha}, & \Omega_{w}=\xi
\end{array}
$$

(точный результат). Эта фиксированная точка соответствует новому нетривиальному ИК-скейлинговому режиму (классу универсальности), в котором нелинейность модели (2.4) и турбулентное перемешивание важны одновременно.

На рис. 3 показаны области ИК-устойчивости для всех фиксированных точек на плоскости $(\varepsilon, \xi)$ : темная область для гауссовой точки, горизонтальные полосы для точки модели КПЖ, вертикальные полосы для точки модели Крейчнана и светло-серая область для нового режима. В этих областях оба собственных числа матрицы (5.2) для данных фиксированных точек положительны. 


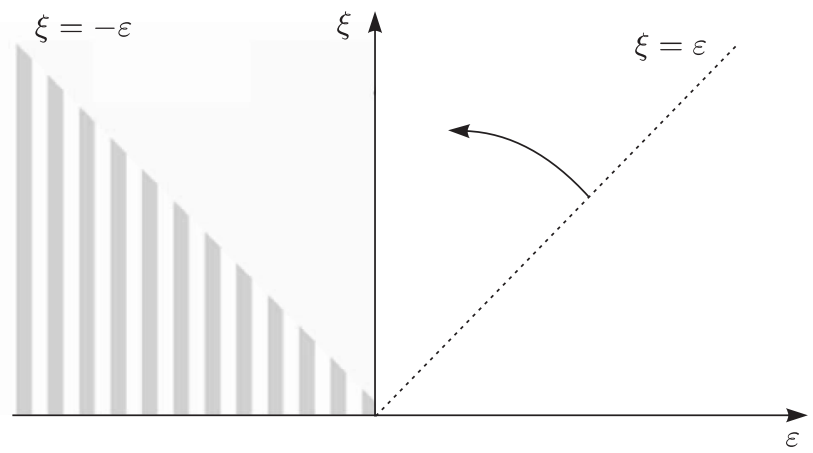

Рис. 4. Граница между областями 3 и 4 движется против часовой стрелки с ростом сжимаемости $\alpha$, пока не достигнет луча $\varepsilon=-\xi$ (для $d=2$ ).

В однопетлевом приближении (5.3) все границы областей устойчивости - прямые линии. Между разными областями нет ни пустот, ни пересечений. Такая картина характерная особенность приближения первого порядка. Границы $\varepsilon<0, \xi<0$ для первой точки, $\varepsilon>0$ для второй точки и $\xi>0$ для третьей точки точные, тогда как на остальные могут повлиять поправки старших порядков, т.е. границы могут искривляться, а между разными областями ИК-устойчивости могут появляться пустоты и пересечения.

Главное качественное заключение, которое можно сделать из этой схемы, состоит в том, что для малых $\alpha$ и наиболее реалистичных значений $d=1$ или $d=2$ и $\xi=4 / 3$ или $\xi=2$ ИК-асимптотическое поведение управляется фиксированной точкой модели Крейчнана. Однако, по мере того как степень сжимаемости $\alpha$ возрастает, область устойчивости новой точки становится шире и, наконец, поглощает реалистичные значения $\varepsilon$ и $\xi$. Действительно, граница между областями 3 и 4 зависит от $\alpha$. Когда $\alpha$ растет, граница вращается против часовой стрелки и при $\alpha \rightarrow \infty$ приближается к лучу $\varepsilon=(1-d) \xi$. Таким образом, для $d=1$ она стремится к вертикальному лучу $\varepsilon=0, \xi>0$ а для $d=2$ стремится к $\varepsilon=-\xi, \xi>0$ (см. рис. 4). Заметим, что для $d=2$ граница становится вертикальной при $\alpha=1$.

Следует помнить, однако, что результаты, связанные с границей между областями 3 и 4, могут быть подвержены влиянию вкладов старших порядков. Стоит также отметить, что интерпретация четвертой фиксированной точки приводит к той же проблеме, что и интерпретация точки модели КПЖ: когда она является ИК-притягивающей, координата $g_{*}$ лежит в нефизической области, $g_{*}<0$. Мы вернемся к этой проблеме в разделе 7.

\section{6. КРИТИЧЕСКИЕ РАЗМЕРНОСТИ. КРИТИЧЕСКИЙ СКЕЙЛИНГ СТРУКТУРНЫХ ФУНКЦИЙ. СОСТАВНЫЕ ПОЛЯ $h^{n}(x)$.}

Наличие ИК-притягивающей точки свидетельствует о существовании скейлингого (самоподобного) поведения функций Грина в ИК-области. В этом критическом скейлинге все "ИК-несущественные" параметры ( $\mu, g$ и $w$ в нашем случае) фиксируются, тогда как "ИК-существенные" параметры (координаты/импульсы, время/частоты) растягиваются. Критическая размерность $\Delta_{F}$ некоторой ИК-суще- 
ственной величины $F$ дается соотношением (с нормировочным условием $\Delta_{k}=1$ )

$$
\Delta_{F}=d_{F}^{k}+\Delta_{\omega} d_{F}^{\omega}+\gamma_{F}^{*},
$$

где $\Delta_{\omega}=2-\gamma_{\varkappa}^{*}$ есть критическая размерность частоты, $d_{F}^{k, \omega}-$ канонические размерности величины $F$, заданные в табл. 1 , и $\gamma_{F}^{*}$ - значение аномальной размерности (4.3) в обсуждаемой фиксированной точке: $\gamma_{F}^{*}=\gamma_{F}\left(g_{*}, w_{*}\right)$ (см., например, монографию [11] для детального объяснения).

Из табл. 1 для размерностей полей мы находим

$$
\Delta_{h}=-2+\Delta_{\omega}+\gamma_{h}^{*}, \quad \Delta_{h^{\prime}}=(d+2)-\Delta_{\omega}+\gamma_{h}^{*} .
$$

Тогда соотношения (4.6) дают

$$
\Delta_{\omega}=2-\gamma_{2}^{*}, \quad \Delta_{h}=-\gamma_{2}^{*}+\gamma_{3}^{*}, \quad \Delta_{h^{\prime}}=d+\gamma_{2}^{*}-\gamma_{3}^{*} .
$$

Наконец, подставляя явные однопетлевые выражения (4.8), находим, что для первой и второй фиксированных точек

$$
\Delta_{h}=0, \quad \Delta_{h^{\prime}}=d, \quad \Delta_{\omega}=2,
$$

а для третьей и четвертой фиксированных точек

$$
\Delta_{h}=0, \quad \Delta_{h^{\prime}}=d, \quad \Delta_{\omega}=2-\xi .
$$

Для точек 1-3 эти выражения точные (см. замечание после уравнения (5.4)). Для четвертой точки размерности полей могут меняться из-за вкладов старших порядков при сохранении точного условия $\Delta_{h}+\Delta_{h^{\prime}}=d$, а выражение для $\Delta_{\omega}$ точное вследствие второго соотношения в (5.3).

В традиционных обозначениях (1.1) $\Delta_{h}=-\chi$ и $\Delta_{\omega}=z$. Однако величина $S_{n}$ в $(1.1)$ - это не обычная функция Грина порядка $n$ основных полей $h(x)$ : это сумма функций парных корреляторов $\left\langle h^{s}(x) h^{q}(0)\right\rangle$ составных полей ("составных операторов" в теоретико-полевой терминологии) $h^{n}(x)$. Перенормировка таких величин требует дальнейшего анализа, но в настоящем случае он весьма прост.

В нашей модели полная каноническая размерность 1-неприводимой функции Грина $\Gamma=\langle F \Phi \ldots \Phi\rangle_{1 \text {-непр }}$ с одним составным оператором $F$ и произвольным числом базовых полей $\Phi=\left\{h, h^{\prime}, v\right\}$ равна $d_{\Gamma}=d_{F}-d_{h} N_{h}-d_{h^{\prime}} N_{h^{\prime}}-d_{v} N_{v}$, где $N_{h}, N_{h^{\prime}}, N_{v}-$ числа соответствующих полей, входящих в функцию $\Gamma$, a $d_{h}, d_{h^{\prime}}, d_{v}-$ их канонические размерности и $d_{F}$ - каноническая размерность $F$ (см., например, книгу [11]). Формальный индекс расходимости есть $\delta_{\Gamma}=\left.d_{\Gamma}\right|_{\varepsilon=\xi=0}$; поверхностные расходимости могут присутствовать в $\Gamma$, если $\delta_{\Gamma}-$ неотрицательное целое число.

Из табл. 1 для $F=h^{n}$ находим, что $d_{F}=0$ и $\delta_{\Gamma}=-2 N_{h^{\prime}}-N_{v}$. Таким образом, расходимости, на первый взгляд, могут присутствовать во всех функциях

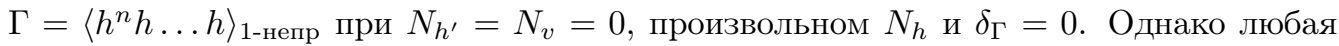
нетривиальная диаграмма такой функции содержит хотя бы одну внешнюю вершину $h^{\prime}(\partial h)^{2}$ или $h^{\prime}(v \partial) h$, где поле $h$ входит под знаком производной. Таким образом, по крайней мере один внешний импульс возникает в диаграмме как общий множитель, реальный индекс $\delta_{\Gamma}^{\prime}$ отрицателен, и поверхностные расходимости на самом деле отсутствуют. Это означает, что все операторы $F=h^{n}$ не перенормируются, и их критические размерности просто даются выражением $\Delta_{F}=n \Delta_{h}$. Это дает обоснование соотношения (1.1) с размерностями (6.3), (6.4). 


\section{7. ОБСУЖДЕНИЕ И ЗАКЛЮЧЕНИЕ}

Мы изучили эффекты турбулентного перемешивания в задаче случайного роста границы раздела фаз. Рост моделировался хорошо известным стохастическим уравнением КПЖ (1.2), (1.3). Турбулентное поле скорости моделировалось ансамблем "быстрых изменений" Крейчнана (1.5).

Задача в целом может быть сформулирована как мультипликативно перенормируемая модель с функционалом действия (2.4). Используя теоретико-полевую РГ, мы показали, что в зависимости от отношения между критическим индексом $\xi$ и пространственной размерностью $d$ система демонстрирует различные типы ИК-поведения, связанного с четырьмя возможными фиксированными точками уравнений РГ. В дополнение к известным режимам (обыкновенная диффузия, обыкновенный процесс роста и пассивное адвективное скалярное поле) установлено существование нового неравновесного класса универсальности.

Практические вычисления координат фиксированных точек, их областей устойчивости и критических размерностей были выполнены в первом порядке двойного разложения по $\xi$ и $\varepsilon=2-d$ (однопетлевое приближение). Было показано, что для несжимаемой жидкости наиболее реалистичные значения $\xi$ и $d$ относятся к классу универсальности пассивного скалярного поля, в котором нелинейность модели КПЖ несущественна. Если степень сжимаемости $\alpha$ становится достаточно большой, то происходит смена типа ИК-поведения, и значения $d$ и $\xi$ попадают в область устойчивости нового режима.

Тем не менее некоторые вопросы остаются без ответа. В новом классе универсальности (как и для модели КПЖ) координаты фиксированной точки лежат в нефизичной области $g_{*}<0$, которая отвечает "неправильному" отрицательному знаку амплитуды парного коррелятора (1.3). Таким образом, данный класс универсальности требует аккуратной физической интерпретации. В этой связи можно вспомнить, что в формализме Дои-Пелити [43], [44], где исходная микроскопическая задача формулируется в терминах операторов рождения и уничтожения, члены, квадратичные по полям отклика, могут появляться в функционалах действия с отрицательными знаками [44].

Другой вопрос - судьба сильно взаимодействующей фиксированной точки чистой модели КПЖ [24]. Если она действительно существует, то определенно сохраняется в нашей задаче (в ситуации $w_{*}=0$ ), но может стать неустойчивой. С другой стороны, могут возникать непертурбативные фиксированные точки с $w_{*} \neq 0$.

В нашем анализе мы использовали простейший ансамбль Крейчнана для адвективного поля скорости. Было бы интересно рассмотреть более реалистичные модели: негауссовы, с конечным временем корреляции, анизотропией и т. п. Эта работа ведется в настоящий момент.

\section{ПРИЛОЖЕНИЕ}

\section{Вычисление перенормировочных констант}

В этом приложении мы кратко излагаем вывод полученных результатов для перенормировочных констант (3.10) в первом порядке. Хотя однопетлевое вычисление достаточно простое и может быть проведено различными способами, его стоит обсудить для полноты изложения и для того, чтобы продемонстрировать некоторые интересные тонкости, специфические для рассматриваемой модели (2.4). 
Перенормировочные константы определяются требованием, чтобы функции Грина перенормированной модели (3.6), выраженные в перенормированных переменных, были УФ-конечны (в нашем случае были конечны при $\varepsilon \rightarrow 0, \xi \rightarrow 0$ ). Полный

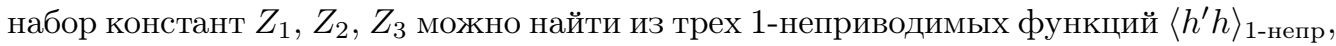

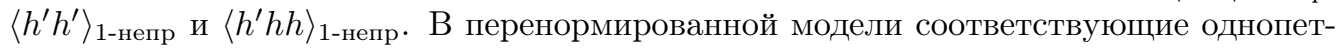
левые приближения имеют вид

$$
\begin{aligned}
& \left\langle h^{\prime} h\right\rangle_{1 \text {-непр }}=\mathrm{i} \eta-\varkappa p^{2} Z_{2}+\smile \bigcirc-+\smile, \\
& \left\langle h^{\prime} h^{\prime}\right\rangle_{1 \text {-непр }}=D Z_{1}+\frac{1}{2}=+\bigcirc-+\longleftarrow
\end{aligned}
$$

и

$$
\begin{aligned}
\left\langle h^{\prime} h h\right\rangle_{1-\text { непр }}=\quad & +\Delta++\Delta+ \\
& +\Delta+\Delta+\Delta .
\end{aligned}
$$

Здесь мы обозначили затравочный пропагатор $\langle h h\rangle_{0}$ прямой линией, $\left\langle h h^{\prime}\right\rangle_{0}-$ прямой линией со штрихом, отвечающим полю $h^{\prime}$, и пропагатор скорости - волнистой линией. В дальнейшем мы полагаем внешнюю частоту $\eta$ равной нулю, так как рас-

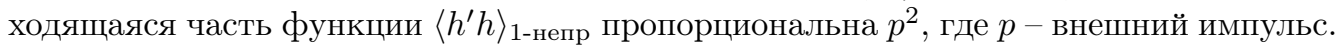

Все диаграммные элементы нужно выразить в перенормированных переменных, используя соотношения (3.6)-(3.9). В однопетлевом приближении константы $Z_{i}$ в затравочных членах (П.1)-(П.3) должны быть взяты в первом порядке по $g$ и $w$, тогда как в однопетлевых вкладах их нужно просто заменить на единицы, $Z_{i} \rightarrow 1$. Таким образом, переход к перенормированным переменным в однопетлевых диаграммах достигается за счет простой замены $\varkappa_{0} \rightarrow \varkappa, g_{0} \rightarrow g \mu^{\varepsilon}$ и $w_{0} \rightarrow w \mu^{\xi}$.

ИК-регуляризация в диаграммах, включающая пропагатор скорости, обеспечивается обрезанием в интеграле (1.5) при $k=m$. В остальных диаграммах она обеспечивается внешними импульсами и частотами. Нас интересуют, однако, только УФ-расходящиеся части этих диаграмм (полюсы по $\varepsilon$ и $\xi$ ). Поэтому мы используем следующий прием, упрощающий вычисления: интегрирование по импульсам во всех диаграммах обрезается снизу на значении $k=m$. Тогда в логарифмически расходящихся функциях (П.2) и (П.3) внешние импульсы и частоты можно приравнять нулю, а в квадратично расходящейся функции (П.1) мы будем удерживать только член порядка $p^{2}$ в разложении по внешнему импульсу $p$.

Теперь легко провести интегрирование по частоте. Возникающие в результате интегралы по импульсам с помощью формул

$$
\int d \mathbf{k} k_{i} f(k)=0, \quad \int d \mathbf{k} \frac{k_{i} k_{s}}{k^{2}} f(k)=\frac{\delta_{i s}}{d} \int d \mathbf{k} f(k),
$$

где $f(k)$ - произвольная функция, зависящая только от $k=|\mathbf{k}|$, сводятся к скалярному интегралу

$$
J(m)=\int_{k>m} d \mathbf{k} \frac{1}{k^{d+y}}=S_{d} \frac{m^{-y}}{y},
$$

где $S_{d}=2 \pi^{d} / \Gamma(d / 2)$ - площадь поверхности единичной сферы в пространстве размерности $d$ и $y=\varepsilon$ либо $y=\xi$.

Последние две диаграммы в (П.3) на самом деле исчезают и, таким образом, не дают вклада в перенормировочную константу. Действительно, они содержат замкнутые циклы запаздывающих пропагаторов; при этом принципиальное значение 
имеет тот факт, что коррелятор скорости содержит дельта-функцию по времени. Простое вычисление показывает, что первые три диаграммы в (П.3) также не дают вклада в $Z_{3}$, так как их расходящиеся части взаимно сокращают друг друга. Это следствие галилеевой симметрии (3.4) исходной модели КПЖ, которая запрещает появление контрчленов типа $h^{\prime}(\partial h)^{2}$ во всех порядках теории возмущений.

Аналитическое выражение для оставшейся диаграммы в (П.3) выглядит следующим образом:

$$
p_{i} p_{j} \int \frac{d \omega}{2 \pi} \int_{k>m} \frac{d \mathbf{k}}{(2 \pi)^{d}} \frac{k^{2}}{\omega^{2}+\varkappa^{2} k^{4}} \frac{w \varkappa \mu^{\xi}}{k^{d+\xi}}\left\{P_{i j}(\mathbf{k})+\alpha Q_{i j}(\mathbf{k})\right\} .
$$

Здесь множитель перед интегралом возникает из двух нижних вершин, первый множитель в интеграле - из верхней вершины (в числителе) и пропагаторов $\left\langle h^{\prime} h\right\rangle_{0}$ (в знаменателе), последний сомножитель - коррелятор скорости. Выполнив вычисление, как описано выше, мы окончательно получаем для (П.3) выражение

$$
\left\langle h^{\prime} h h\right\rangle_{1 \text {-непр }}=p^{2}\left\{Z_{3}+\frac{\hat{w}}{\xi}\left(\frac{\mu}{m}\right)^{\xi} \frac{d-1+\alpha}{2 d}\right\} .
$$

Множитель $(\mu / m)^{\xi}$ УФ-конечен: он стремится к единице при $\xi \rightarrow 0$. Мы можем видеть, что для устранения полюса по $\xi$ в (П.7) перенормировочную константу $Z_{3}$ действительно можно выбрать в виде, заданном в (3.10).

Теперь обратимся к перенормировочной константе $Z_{2}$. Первая из двух диаграмм в (П.1) оказывается УФ-конечной и не дает вклада в $Z_{2}$. Чтобы в этом убедиться, можно рассмотреть следующее аналитическое выражение (с точностью до несущественных амплитудных множителей):

$$
\begin{aligned}
\int \frac{d \omega}{2 \pi} & \int_{k>m} \frac{d \mathbf{k}}{(2 \pi)^{d}} \frac{k_{i}(p+k)_{i} k_{j} p_{j}}{\omega^{2}+\varkappa^{2} k^{4}} \frac{1}{-\mathrm{i} \omega+\varkappa|\mathbf{k}+\mathbf{p}|^{2}} \propto \\
& \propto \int_{k>m} \frac{d \mathbf{k}}{(2 \pi)^{d}} \frac{k_{i}(p+k)_{i} k_{j} p_{j}}{k^{2}\left(k^{2}+|\mathbf{k}+\mathbf{p}|^{2}\right)}
\end{aligned}
$$

Нас интересует член типа $p^{2}$ в разложении по $p$. Он оказывается равным сумме двух одинаковых интегралов с противоположными знаками. Действительно, первый из этих интегралов возникает от вклада $p_{i} p_{j} k_{i} k_{j}$ в числителе; тогда в знаменателе мы можем положить $\mathbf{p}=0$. Возникающее подынтегральное выражение становится равным $p_{i} p_{j} k_{i} k_{j} / 2 k^{4}$. Второй интеграл возникает от вклада $k_{i} k_{i} k_{j} p_{j}$. Тогда нужно разлагать знаменатель до членов порядка $\mathcal{O}(\mathbf{p})$ :

$$
\int_{k>m} \frac{d \mathbf{k}}{(2 \pi)^{d}} \frac{k_{j} p_{j}}{k^{2}+|\mathbf{k}+\mathbf{p}|^{2}} \simeq \int_{k>m} \frac{d \mathbf{k}}{(2 \pi)^{d}} \frac{k_{j} p_{j}}{2 k^{2}}\left\{1-\frac{(\mathbf{p k})}{k^{2}}\right\} .
$$

Первое слагаемое в правой части (П.9) исчезает ввиду нечетности подынтегральной функции по k, а второе равно интегралу, вычисленному выше, но с другим знаком.

Аналитическое выражение для второй диаграммы в (П.1) следующее:

$$
\int \frac{d \omega}{2 \pi} \int_{k>m} \frac{d \mathbf{k}}{(2 \pi)^{d}} \frac{\mathrm{i} p_{i} \mathrm{i}(p+k)_{j}}{k^{d+\xi}} w \varkappa \mu^{\xi} \frac{P_{i j}(\mathbf{k})+\alpha Q_{i j}(\mathbf{k})}{-\mathrm{i} \omega+\varkappa|\mathbf{k}+\mathbf{p}|^{2}} .
$$

Интегрирование по $\omega$ содержит неопределенность:

$$
\int \frac{d \omega}{2 \pi} \frac{1}{-\mathrm{i} \omega+\varkappa|\mathbf{k}+\mathbf{p}|^{2}}=\theta(0),
$$




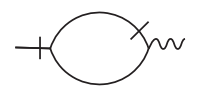

Рис. 5. Однопетлевой вклад в функцию $\left\langle h^{\prime} v\right\rangle_{1-\text {-непр }}$.

где $\theta(0)$ - функция Хевисайда в нуле. Она отражает детали статистики поля скорости, потерянные в пределе белого шума (см. обсуждение в работе [37]). В нашем случае дельта-функцию в (1.5) следует понимать как предел некоторой "узкой” функции, которая обязательно симметрична по $t, t^{\prime}$, так как мы имеем дело с парным коррелятором. Таким образом, неопределенность в (П.11) должна быть недвусмысленно разрешена как сумма пределов: $\theta(0)=1 / 2$. Подынтегральная функция возникающего в итоге интеграла по $\mathbf{k}$ в (П.10) имеет нечетную часть, которую можно отбросить, и четную, которая дает

$$
\left\langle h^{\prime} h\right\rangle_{1 \text {-непр }}=-\varkappa p^{2}\left\{Z_{2}+\frac{\hat{w}}{\xi}\left(\frac{\mu}{m}\right)^{\xi} \frac{d-1+\alpha}{2 d}\right\} .
$$

Мы видим, что $Z_{2}$ можно выбрать в виде (3.10); в однопетлевом приближении получается $Z_{2}=Z_{3}$.

Мы не будем детально обсуждать вычисление $Z_{1}$ : первая диаграмма сводится к скалярному интегралу (П.5) с $y=\varepsilon$ сразу после интегрирования по частоте. Аналитическое выражение для второй диаграммы аналогично (П.6) с точностью до замены $p_{i} p_{j} \rightarrow k_{i} k_{j}$, так что выживает только вклад от продольного проектора. Это приводит к замене $(d-1+\alpha) / 2 d \rightarrow \alpha$.

Собирая все вклады вместе и учитывая симметрийный коэффициент $1 / 2$ для первой диаграммы, получаем

$$
\left\langle h^{\prime} h^{\prime}\right\rangle_{1 \text {-непр }}=D\left\{Z_{1}+\frac{1}{8} \frac{\hat{g}}{\varepsilon}\left(\frac{\mu}{m}\right)^{\varepsilon}+\alpha \frac{\hat{w}}{2 \xi}\left(\frac{\mu}{m}\right)^{\xi}\right\} .
$$

Мы видим, что константу $Z_{1}$, удаляющую полюсы из выражения (П.13), можно взять из (3.10).

Остается только обсудить возможные УФ-расходимости в функции $\left\langle h^{\prime} v\right\rangle_{1 \text {-непр }}$ с контрчленом $h^{\prime} \partial_{i} v_{i}$. Единственная однопетлевая диаграмма для этой функции показана на рис. 5. Несложно убедиться в том, что соответствующее аналитическое выражение почти идентично тому, которое получилось для первой диаграммы в функции (П.1): последняя отличается только дополнительным множителем $p_{j}$. Тем самым обсуждаемая диаграмма также УФ-конечна и не вызывает появления соответствующего контрчлена.

Благодарности. Авторы выражают признательность Л. Ц. Аджемяну за полезные обсуждения. Авторы также благодарны Санкт-Петербургскому государственному университету за финансовую поддержку в рамках исследовательского гранта 11.38.185.2014.

\section{Список литературы}

[1] J. Krug, H. Spohn, "Kinetic roughening of growing surfaces", Solids Far From Equilibrium: Growth, Morphology, and Defects, ed. C. Godréche, Cambridge Univ. Press, Cambridge, 1990, 479-582.

[2] T. Halpin-Healy, Y.-C. Zhang, Phys. Rep., 254:4 (1995), 215-414. 
[3] A.-L. Barabási, H. E. Stanley, Fractal Concepts in Surface Growth, Cambridge Univ. Press, Cambridge, 1995.

[4] J. Krug, Adv. Phys., 46:2 (1997), 139-282.

[5] M. Lässig, J. Phys.: Cond. Matter, 10:44 (1998), 9905-9950, arXiv: cond-mat/9806330.

[6] M. Eden, "A Two-dimensional growth process", Berkeley Symposium on Mathematical Statistics and Probability, v. 4: Contributions to Biology and Problems of Medicine, ed. J. Neyman, Cambridge Univ. Press, Cambridge, 1961, 223-239.

[7] S. F. Edwards, D. R. Wilkinson, Proc. R. Soc. London Ser. A, 381:1780 (1982), 17-31.

[8] J. M. Kim, J. M. Kosterlitz, T. Ala-Nissila, J. Phys. A: Math. Gen., 24:23 (1991), $5569-5586$.

[9] M. D. Penrose, J. Stat. Phys., 131:2 (2008), 247-268.

[10] J. Zinn-Justin, Quantum Field Theory and Critical Phenomena, Clarendon Press, Oxford, 1989.

[11] А.Н. Васильев, Квантовополевая ренормгруппа в теории критического поведения и стохастической динамике, ПИЯФ, СПб., 1998.

[12] M. Kardar, G. Parisi, Y.-C. Zhang, Phys. Rev. Lett., 56:9 (1986), 889-92.

[13] D. Forster, D. R. Nelson, M. J. Stephen, Phys. Rev. A, 16:2 (1977), 732-749.

[14] M. Kardar, Y.-C. Zhang, Phys. Rev. Lett., 58:20 (1987), 2087-2090; J. P. Bouchaud, M. Mézard, G. Parisi, Phys. Rev. E, 52:4 (1995), 3656-3674, arXiv: cond-mat/9503144; E. Frey, U.C. Täuber, T. Hwa, Phys. Rev. E, 53:5 (1996), 4424-4438, arXiv: cond-mat/9601049.

[15] T. Goldman, D. Hochberg, R. Laflamme, J. Pérez-Mercader, Phys. Lett. A, 222:3 (1996), 177-181; J. F. Barbero, A. Domínguez, T. Goldman, J. Pérez-Mercader, Europhys. Lett., 38:8 (1997), 637-642; T. Buchert, A. Domínguez, J. Pérez-Mercader, Astron. Astrophys., 349:2 (1999), 343-353; J. Gaite, A. Domínguez, J. Phys. A: Math. Theor., 40:25 (2007), $6849-6857$.

[16] E. Medina, T. Hwa, M. Kardar, Y.-C. Zhang, Phys. Rev. A, 39:6 (1989), 3053-3075; C.-H. Lam, L. M. Sander, D. E. Wolf, Phys. Rev. A, 46:10 (1992), R6128-R6131.

[17] J. P. Doherty, M. A. Moore, J. M. Kim, A. J. Bray, Phys. Rev. Lett., 72:13 (1994), 2041-2044; M. Kardar, A. Zee, Nucl. Phys. B, 464:3 (1996), 449-462, arXiv: cond-mat/ 9507112; Л. В. Борк, С. Л. Огарков, ТМФ, 178:3 (2014), 416-432.

[18] С. И. Павлик, ЖЭТФ, 106:2 (1994), 553-559; Н. В. Антонов, Ал. Н. Васильев, ЖЖЭТФ, 108:3 (1995), 885-893.

[19] H. Jeong, B. Kahng, D. Kim, Phys. Rev. Lett., 77:25 (1996), 5094-5097, arXiv: cond-mat/9606205; H.-J. Kim, I.-M. Kim, J. M. Kim, Phys. Rev. E, 58:1 (1998), 1144-1147.

[20] T. Hwa, M. Kardar, Phys. Rev. Lett., 62:16 (1989), 1813-1816; Phys. Rev. A, 45:10 (1992), 7002-7023; B. Tadić, Phys. Rev. E, 58:1 (1998), 168-173, arXiv: cond-mat/9912248.

[21] E. Frey, U. C. Täuber, Phys. Rev. E, 50:2 (1994), 1024-1045, arXiv: cond-mat/9406068.

[22] M. Lässig, Nucl. Phys. B, 448:3 (1995), 559-574, arXiv: cond-mat/9501094; K. J. Wiese, J. Stat. Phys., 93:1-2 (1998), 143-154, arXiv: cond-mat/9802068.

[23] M. Lässig, Phys. Rev. Lett., 80:11 (1998), 2366-2369, arXiv: cond-mat/9711037.

[24] L. Canet, H. Chaté, B. Delamotte, N. Wschebor, Phys. Rev. Lett., 104:15 (2010), 150601, 4 pp., arXiv: 0905.1025.

[25] T. Kloss, L. Canet, N. Wschebor, Phys. Rev. E, 86:5 (2012), 051124, 19 pp., arXiv: 1209.4650 .

[26] M. Lässig, H. Kinzelbach, Phys. Rev. Lett., 78:5 (1997), 903-906.

[27] M. Hairer, Ann. Math., 178:2 (2013), 559-664.

[28] Д. Ю. Иванов, Критическое поведение неидеализированных систем, Физматлит, М., 2013. 
[29] В. В. Прудников, П. В. Прудников, А. Н. Вакилов, Теоретико-полевые и численные методы описания критических явлений в структурно неупорядоченных системах, Омский ГУ, Омск, 2012.

[30] G. Satten, D. Ronis, Phys. Rev. Lett., 55:1 (1985), 91-94; Phys. Rev. A, 33:5 (1986), $3415-3432$.

[31] A. Onuki, K. Kawasaki, Progr. Theoret. Phys., 63:1 (1980), 122-139; A. Onukiand, K. Yamasaki, K. Kawasaki, Ann. Phys., 131:1 (1981), 217-242; T. Imaeda, A. Onuki, K. Kawasaki, Progr. Theor. Phys., 71:1 (1984), 16-26.

[32] D. Beysens, M. Gbadamassi, L. Boyer, Phys. Rev. Lett., 43:17 (1979), 1253-1256; D. Beysens, M. Gbadamassi, J. Physique Lett., 40:21 (1979), 565-567.

[33] R. Ruiz, D. R. Nelson, Phys. Rev. A, 23:6 (1981), 3224-3246; 24:5 (1981), 2727-2734; A. Aronovitz, D. R. Nelson, Phys. Rev. A, 29:44 (1984), 2012.

[34] N. V. Antonov, M. Hnatich, J. Honkonen, J. Phys. A: Math. Gen., 39:25 (2006), 7867-7887, arXiv: cond-mat/0604434.

[35] N. V. Antonov, A. A. Ignatieva, J. Phys. A: Math. Gen., 39:44 (2006), 13593-13620, arXiv: cond-mat/0607019.

[36] N. V. Antonov, V. I. Iglovikov, A.S. Kapustin, J. Phys. A: Math. Theor., 42:13 (2009), 135001, arXiv: 0808.0076; N. V. Antonov, A. S. Kapustin, J. Phys. A: Math. Theor., 43:40 (2010), 405001; Н. В. Антонов, А. С. Капустин, А. В. Малышев, ТМФ, 169:1 (2011), 124-136.

[37] G. Falkovich, K. Gawȩdzki, M. Vergassola, Rev. Modern Phys., 73:4 (2001), 913-975, arXiv: cond-mat/0105199.

[38] N. V. Antonov, J. Phys. A: Math. Gen., 39:25 (2006), 7825-7865.

[39] Д. Ю. Волченков, М. Ю. Налимов, ТМФ, 106:3 (1996), 375-389.

[40] N. V. Antonov, M. M. Kostenko, Phys. Rev. E, 90:6 (2014), 063016, 21 pp., arXiv: 1410.1262 .

[41] P. C. Martin, E. D. Siggia, H. A. Rose, Phys. Rev. A, 8:1 (1973), 423-437.

[42] K. J. Wiese, Phys. Rev. E, 56:5 (1997), 5013-5017, arXiv: cond-mat/9706009.

[43] M. Doi, J. Phys. A: Math. Gen., 9:9 (1976), 1479-1495; P. Grassberger, P. Scheunert, Fortschr. Phys., 28:10 (1980), 547-578; L. Peliti, J. Phys. France, 46:9 (1984), 1469-1483.

[44] U.C. Täuber, Acta Physica Slovaca, 52:6 (2002), 505-513; "Scale invariance and dynamic phase transitions in diffusion-limited reactions", Advances in Solid State Physics, 43, ed. R. Haug, Springer, Berlin, 2003, 659-676; "Field-theory approaches to nonequilibrium dynamics", Ageing and the Glass Transition, Lecture Notes in Physics, 716, eds. M. Henkel, M. Pleimling, R. Sanctuary, Springer, Berlin, 2007, 295-348; "Field theoretic methods", Encyclopedia of Complexity and System Science, ed. R. A. Meyers, Springer, New York, 2009, 3360-3374, arXiv: 0707.0794. 